\title{
BIBLIOGRAFIA SOBRE OLIMPISME
}

\author{
Pilar Cid Leal, Dolors Aparicio Granero \\ Documentalistes del Centre d'Estudis Olimpics \\ de la Universitat Autònoma de Barcelona
}

La selecció següent és una petita mostra de les monografies i dels títols de publicacions periòdiques existents sobre Olimpisme i Barcelona '92. Totes les obres senyalades amb un asterisc han estat revisades personalment per les autores i es poden trobar en algun dels Centres especialitzats més irmportants de Catalunya:

- Centre d'Estudis Olímpics (Universitat Autònoma de Barcelona, Edifici B, Bellaterra)

- Biblioteca de l'Esport (València 3-5-7)

- INEFC Barcelona (Ávinguda de l'Estadi s/n, Anella Olímpica)

— INEF Lleida (Pl. Caparrella $s / n$, Lleida)

\section{MONOGRAFIES}

\section{OBRES DE REFERENCIA}

\subsection{Bibliografies}

* Bibliographie des oeuvres du Baron Pierre de Coubertin = Bibliography of the works of Baron Pierre de Coubertin. - [S.I. : s.n.], 1968. - 16 p.

\section{Ghent, G.}

Olympic bibliography : guide to sources of information for the olympic games with special emphasis on the winter games. - 2nd. ed. - Calgary : University of Calgary. Social Sciences Library, 1984. - 1 v. (paginació múltiple)

* Mallon, Bill

The Olympics : a bibliography / Bill Mallon. - New York [etc.] : Garland, 1984. -258 p. - (Garland reference library of social science ; 246) 
Mitra, G.

Constelatia olimpiadelor : lexicon olimpic/G. Mitra, Retinschi. - Bucuresti : Sport Turism, $1984 .-487 \mathrm{p}$.

The Olympics : a social science perspective. - Waterloo : SIRLS : University of Waterloo, 1984. - $92 \mathrm{p}$.

Paul, R.

Bibliography of articles published in the offtcial proceedings of the general sessions of the International Olympic Academy 1961-1983 / R. Paul. - Colorado Springs : United States Olympic Commitree, [1986]. - 1 v.

Piper, T.

Champions of gold / T. Piper, F. Crook, D. Anderson. - Blue Earth : Pipier, cop. 1984. - I v.

\subsection{Biografies}

Biographies olympiques : membres du $\mathrm{ClO}$ en activite ou honoraires $=$ Olympic biographies : active or honorary members of the IOC. - Lausanne : Comité International Olympique, cop. 1987. - I25 p. : il.

* Brundage, Avery

Memorias / Avery Brundage. - Madrid : Editora Nacional, 1973 - $370 \mathrm{p}$. - (Cultura y deporte). - ISBN 84-276-1 133-1

* Kamper, Erich

Lexikon der 14000 olympioniken : who's who at the olympics / Erich Kamper. - Graz : Leykam, cop. 1983. — XXXV, 687, (136) p. : il. — ISBN 3-7011$7144-0$

Killanin, M.M.

My olympic years / M.M. Killanin. — London : Secker \& Warburg, cop. 1983.

\section{Mallon, Bill} - XVII, 238 p. : il.

Quest for gold : the encyclopedia of american olympians / Bill Mallon, I. Buchanan, J. Tishman. - New York : Leisure Press, cop. 1984. - 495 p. : il.

* Navacelle, Geoffroy de

Pietre de Coubertin : sa vie par l'image / par Geoffroy de Navacelle. - Zürich [etc.] : Weidmann, [1986]. - 96 p.:il. — ISBN 3-296-18004-4

Olympic biographies. — Lausanne : International Olympic Committee, I981. $171 \mathrm{p}$.

* Schöbel, Heinz

The Four dimensions of Avery Brundage / Heinz Schöbel. — Leipzig : Edition Leipzig, cop. 1968. - 194 p. : it.

* Ullrich, Klaus

Coubertin : leben, denken und schaffen eines humanisten / Klaus Ullrich. Berlin : Sportverlag, 1982. - 180 p.: il. 
Bibliografia sobre olimpisme

\subsection{Diccionaris}

* Bañeres, Enric

Diccionari de l'esport català-castellà castellà-català / Enric Bañeress, Manuẹl de Seabra i Ester Bonct. - Barcelona : Enciclopèdia Catalana, cop. 1989. - 408 p. - ISBN 84-7739-072-X

* [Diccionaris dels esports olímpics]. - Barcelona : Enciclopèdia Catalana, 1991.

- Es tracta d'una col.lecció de 28 diccionaris dets esports oficials i de demostració, a més d'un diccionari general dels esports olímpics

Gavrilovets, A.

English-Russian dictionary of sports terms and phrases : summer olympic games and sports = Anglo-Russkiy slovar'-razgovornik : letnie olimpijskie vidu sporta $/$

A. Gavrilovets . - Moscow : Russian Language Publishers, cop. $1979 .-420$ p.

* Multilingual lexicon for universidade sports : english, français, español, deutsch, pyccknñ / R.L. Busch and Hans J. Bergman general editors. - Edmonton : The University of Alberta Press, cop. 1983. — XIX, 316 p. - ISBN 0-88864057-9

* Olympisches sportwörterbuch $=$ Olympic sports dictionary $=$ Dictionnaire des sports olympiques $=$ Diccionario de deportes olímpicos $/$ [herausgeber Robert E. Lembke]. - München : Langenscheidt, 1972. - 319 p.

* Petiot, Georges

Le Robert des sports : dictionnaire de la langue des sports / par Gcorges Petiot.

- Paris : Le Robert, DL 1987. - XXIV, 553 p.- ISBN 2-85036-098-8

Schoenfelder, K.J.

Kleines olympisches lexikon / K.]. Schoenfelder, F. Frogsch. — Leipzig : Bibliographisches Institut, $1980 .-297 \mathrm{p}$.

\subsection{Directoris $i$ Guies}

* European inventory of sports information and documentation centres / edited by Albert Remans. - Brussels : Clearing House, 1990. - $124 \mathrm{p}$.

* Répertoire du mouvement olympique = Olympic movement directory $: 1991$.

- Lausanne : Comité International Olympique, cop. 1991. - 160 p.

* Timmer, Rob

International directory of sports organizations : under the auspices of the In. ternational Association for Sports Information (LASI) / Rob Timmer, Josef Recla. - Haarlem : Uitgeverij de Vrieseborch, cop. 1984. - 429 p. — ISBN 90 6076-197-9

\subsection{Enciclopèdies}

* Enciclopedia de los comités olímpicos nacionales. - Lausana : Comité Olímpico Internacional, cop. 1979-80. - 3 v, : il. 
* La gran enciclopedia de los deportes olímpicos. - Barcelona : Ediciones del Drac, cop. 1989. - 5 v. : il. - ISBN 84-86532-12-4

Kamper, Erich

Encyclopedia of the olympic games / Erich Kamper. - New York : McGraw Hill, 1972. $-360 \mathrm{p}$.

Suzuki, Y.

Encyclopedia of the olympic games / Y. Suzıki. - [S.I.] : Japanese Olympic Committee, $1981 .-868$ p. : il.

\subsection{Records i medalles olimpiques}

\section{* Greenberg, Stan}

The Guiness book of olympics facts and feats / Stan Greenberg. - Enfield : Guiness Superlatives, 1984. - 256 p.

\section{Greenberg, Stan}

Olympic games : the records / S. Greenberg. - New York : Guinness Superlatives, cop. 1987. - 176 p. : il.

* Gueorguiev, Nikolay

Le Programme et les medailles de la natation des jeux de l'olympiade 1896-1988 / recherche élaborke par Nikolay Gueorguiev, - Lausanne : Maison Olympique, 1990. - 162 p. - Edició no comercial

\section{* Gueorguiev, Nikolay}

Le Programme er les medailles de l'athletisme aux jeux de l'olympiade 18961988 / etude élaborée par Nikolay Gueorguiev. - Lausanne : Maison Olympique, 1990. - 201 p. - Edició no comercial

* Gueorguiev, Nikolay

Le Programme, la participation et les medailles de l'aviron aux jeux olympiques 1900-1988 / recherche effectuée par Nikolay Gueorguiev. — Lausanne : Mäison Olympique, 1991. - 132 p. -- Edició no comercial

* Gueorguiev, Nikolay

Le Programme, la participation et les medailles de l'escrime aux jeux olympiques 1896-1988 / recherche effectuée par Nikolay Gueorguiev. — Lausanne : Maison Olympique, 1990 . - 142 p. - Edició no comercial

* Gueorguiev, Nikolay

Le Programme, la participation et les medailles des XV jeux olympiques d'hiver I recherche effectuée pat Nikolay Gueorguiev. - Lausanne : Maison Olympique, 1990. - 109 p. - Edició no comercial

* Gueorguiev, Nikolay

Le Programme, la participation et les medailles des jeux de la XXIV olympiade / recherche effectuée par Nikolay Gueorguiev. - Lausanne : Maison Olympique, $1990 .-96$ p. - Edició no comercial

* Gueorguiev, Nikolay

Le Ptogramme, la participation et les medailles des sports equestres aux jeux 
olympiques 1900-1988 / recherche effectuée par Nikolay Gueorguiev. — Lausanne : Maison Olympique, 115 p. - Edició no comercial

* Gueorguiev, Nikolay

Le Programme, la participation et les medailles du canoe-kayak aux jeux olympiques 1936-1988 / recherche effectuée par Nikolay Gueorguiev. — Lausanne : Maison Olympique, 1991. - 129 p. - Edició no comercial

* Gueorguiev, Nikolay

Le Programme, la participation et les medailles du penthathlon moderne aux jeux olympiques 1912-1988 / recherche effectuée par Nikolay Gueorguiev. -Lausanne : Maison Olympique, 1991. - 64 p. - Edició no comercial

Guinness book of olympic tecords : compete roll of olympic medal winners (18961980) for the 28 sports ( 7 winter and 21 summer) contested in the $1980 \mathrm{ce}$ lebrations and other useful information/S. McWhirter ... [et al.]. - Toronto : Bantam Books, cop. 1983. - X, 26I p.

Hugman, B.J.

The olympics games : complete track and field results 1896-1988 / B.J. Hugman, P. Arnold. - New York : Facts on File, cop. 1988

Mallon, Bill

The olympic record book / Bill Mallon. — New York : Garland, 1988. - XV, $522 \mathrm{p}$.

* Tales of gold / Lewis H. Carison and John J. Fogarty [ed.]. - Chicago [etc.]: Conremporary Books, cop. 1987. - 514 p. : il. — ISBN 0-8092-5067-5

\section{MOVIMENT OLfMPIC}

\subsection{Obres generals}

Binder, D.

Rassemblez-vous a la decouverte de l'olympisme / D. Binder. - Calgary : Comité d'Organisation des XVes Jeux Olympiques d'Hiver, cop. 1987. - I v.

${ }^{*}$ Coubertin, Pierre de

Mémoires olympiques / par Pierre de Coubertin. - Lausanne : Comité International Olympique, cop. 1979. - 141 p. : il.

* Coubertin, Pierre de

The Olympic idea : discourses and essays / Pierre de Coubertin. - Köl : CarlDiem-Institut, cop. 1966. - VIII, 147 p.

* Coubertin Symposium (1986 : Lausana)

L'Actualité de Pierre de Coubertin : rapport du Symposium du 18 au 20 mars 1986 a l'Université de Lausanne $=$ The Relevance of Pierre de Coubertin today : report of the Symposium 18th to 20 th march 1986 at the University of Lausanne / directeur de l'édition Norbert Müller. - Niedernhausen : Schors, 1987. - 312 p. : il. - ISBN 3-88500-239-6

* De Moscou à Lausanne : dix années de la présidence de Juan Antonio Samaranch $=$ From Moscou to Lausanne $:$ Juan Antonio Samaranch ten years as IOC pre- 
«Papersw: Revista de Sociologia

sident. - Lausanne : Comité International Olympique, cop. 1990. - 147 p. : iI. - ISBN 92-9105-003-6

* Espy, Richard

The Politics of the olympic games : with an epilogue 1976-1980 / Richard Espy. — Berkeley [etc.] : University of California Press, cop. 1981. - XIY, 238 p. ISBN 0-520-04395-2

* Fleuridas, Claude

Les Jeux olympiques : aspects historiques institutionnels sociologiques / Claude Fleuridas, Raymond Thomas. - Paris : Revue E.P.S., 1984. - 142 p. - ISBN 2-85258-330-5

Graham, P.J.

Modern olympics / P.J. Graham, H. Ueberhorst. - Cornwall : Leisure press, 1976. - II, $236 \mathrm{p}$.

* Graupera Monar, Ma Hortensia

Olimpismo-política : 100 años de vida en común / $\mathrm{M}^{2}$ Hortensia Graupera Monar. - [Barcelona : s.n.], 1989. - $330 \mathrm{p}$.

Grombach, J. V.

Official 1980 olympic guide : including the complete summer and winter games = 1980 olympic guide : complete sport by sport history with records of every event / J. V. Grombach. - New York : Times Books, cop. 1980. - 351 p.

* Guttmann, Allen

The Games must go on : Avery Brundage and the olympic movement / Allen Guttmann. - New York : Columbia University Press, 1984. - XI, 317 p. Haralampiev, D.

Congres olympiques 1894-1981 / D. Haralampiev, N. Gheorgulev, E. Kamboutrou. - Sofia : Sofia Presse, 1981. - [193] p. : it.

* Hoberman, John

The Olympic crisis : sport, politics and the moral order/John Hoberman. New Rochelle : Aristide D. Caratzas, cop. 1986. - 167 p. : il. - ISBN 0$89241-224-0$

* International Conference on the Olympics and East/West and South/North Cultural Exchange in the World System (1a: $1987:$ Seül)

The Olympics and east/west and south/north cultural exchange : the papers of the First International Conference on the Olympics and East/West and South/North Cultural Exchange in the World System / Kang Shin-pyo, John MacAloon, Roberto DaMatta editors. - [Seoul] : The Institute for Ethnological Studies Hanyang University, [1987?]. - 781, 122, 21 p. - (Hanyang Ethnology Monograph ; 1 )

Os Jogos olimpicos e as suas perspectivas futuras. - Lisboa : Ministerio da Educaçao e Cultura. Direç̧ao-Geral dos Desportos, 1986. - 49 p.

Le Goulven, F.

Les grandes heures des jeux olympiques / F. Le Goulven, G. Delamarre. - Paris : PAC, cop. $1976 .-175$ p. : il. 


\section{Lekarska, $\mathrm{N}$.}

Essays and studies on olympic problems / N. Lekarska. - Sofia : Medicina \& Fizcultura, 1973. - $121 \mathrm{p}$.

Lucas, J.

Modern olympic games / J. Lucas. - Cranbury : A.S. Barnes, cop. 1980. $242 \mathrm{p}$.

* MacAloon, John J.

This great symbol : Pierre de Coubertin and the origins od the modern olympic games / John J. MacAloon. - Chicago [etc.] : The University of Chicago Press, 1981. - 357 p. : il. - ISBN 0-226-50000-4

* Mercé Varela, Andrés

Los Juegos olímpicos : una ilusión universal / Andtés Mercé Varela. - Barcelona : Edicions 62, 1988. - 141 p. : il. - ISBN 84-297-2783-3

* Meyer, Gaston

El Fenómeno olímpico / Gaston Meyer. - Madrid : Comité Olimpico Español, DL 1963. - 240 p. - (Olimpia)

\section{Meyer, Gaston}

Jeux olympiques : vingt sports au microscope / Gaston Meyer. - Paris : Table Ronde, 1964. - $253 \mathrm{p}$.

* Le Mouvement olympique $=$ The Olympic mouvement. - Lausanne : International Olympic Committee, cop. 1987. - 120 p. : il.

* Müller, Norbert

Von Paris bis Baden-Baden : die olympischen kongresse 1894-1981 / Norbert Müller, - Niedernhausen : Schors, 1983. - 220 p. - (Mainzer Studien zur Sportwissenschaft ; 7). - ISBN 3-88500-194-2

\section{Mukherjee, Subhra}

Olympig games / Subhra Mukherjec. — New Delhi : L'autor, 1980. - XII, $200 \mathrm{p}$.

* Mzali, Mohamed

L'Olympisme aujourd'hui / Mohamed Mzali. - Paris : Les Editions Jeune Afrique, cop. $1984 .-127$ p. : il.

* Ohl, Paul E.

La Guerre olympique / Paul E. Ohl. — Paris : Robert Laffont, cop. 1977. $349 \mathrm{p}$.

* Olympic Congress (10è : 1973 : Varna)

Olympic Congress Varna 1973. - Lausanne : Comité International Olympique, [1973?]. - 163 p. : il.

* Olyropic Congress (11 è : 1981 : Baden-Baden)

11th Olympic Congress in Baden-Baden 1981. - Lausanne : International Olympic Committee, cop. 1982. - 3 v. : il.

* The Olympic garnes in transition / Jeffrcy O. Segrave, Donald Chu editors. Champaing : Human Kinetics Books, cop. 1988, - 443 p. - ISBN 0-87322111-7 
* Olympisme et sport des travailleurs : de Moscou à Los Angeles : progressons ensemble / [realisée par un collectif de dirigeants nationaux de la F.S.G.T.]. Paris : Sport et Plein Air, 1981. - 111 p. : il.

* L'Olympisme par l'affiche : 1896-1984 = Olympism through posters : 1896-1984. - Lausanne : Comité International Olympique, cop. $1983 .-189$ p. : il.

* Pierre de Coubertin : textes choisis. - Zürich [etc.] : Weidmann, 1986. - 3 v. : il.

1: Revelation/ introduction générale choix et présentation des textes: Georges Rioux. - XIV, 666 p. - ISBN 3-296-18001-X

2: Olympisme / introduction, choix et présentation des textes : Norbert Müller. - 760 p. - ISBN 3-296-18002-8

3: Pratique sportive / introduction, choix et présentation des textes : Norbert Müller et Otto Schantz. - 836 p. - ISBN 3-296-18003-6

\section{Piskarev, S.A.}

Na olimpiiskoi oreite : Sbornik/ S.A. Piskarev. - Moskva : Sov. Rossiia, 1980.

$-110 \mathrm{p}$.

Powell, J.T.

Olympics as a human arena / J.T. Powell. - Ease Stroudsburg : East Stroudsburg State College, 1972 . - $14 \mathrm{p}$.

* Simposi Internacional sobre Jocs Olímpics, Comunicació i Intercanvis Culturals (1991 : Barcelona)

[Simposi Internacional sobre Jocs Olímpics, Comunicació i Intercanvis Culturals : l'expertència dels últims quatre jocs olímpics d'estiu. Barcelona, 35/IV/1991]. (Monografia en vies de publicació)

Solakov, A.

New climate in the olympic movement / A. Solakov. - Softa : Softa press, 1976. $-103 \mathrm{p}$.

Solakov, A.

Problems of the olympic movement studies / A. Solakov. - Sofia : BOC, 1976. $-219 \mathrm{p}$.

* Solidarité olympique rapport 1985 = Olympic solidarity report 1985. - Lat1sanne : Solidarité Olympique, [1985]. - 304 p. : il.

United States Olympic Academy. Session (9à : $1985:$ Plattsburgh)

Olympism : a commitment to a better world tomorrow through sport : United States Olympic Academy IX, June 26-30, 1985, State University of New York, Plattsburgh, new York 12901. - [S.I.] : United States Olympic Committee. Education Council, [1985?]. - 253 p.

Vos-Strache, $\mathrm{C}$.

Olympic games : ideals and realities / C. Vos-Strache. - [S.1.] : Leisure Press, [1.981?]. $-224 \mathrm{p}$.

Yalouris, $N$.

Olympic games / N. Yalouris, O. Szymiczek. - Athens : Ekdotike Athenon, cop. $1976 .-495$ p. 
Zilcov, V. A.

Na stupnich virezu : sportovci socialistickych zemi na olympij skych hrach / V.

A. Zilcov. - Moskva : Olympia Fizkultura i Sport, 1980 . - 228 p. : il.

\subsection{Organismes}

* Academia Internacional Olímpica. Sesión (28à : 1988 : Atenes)

Rapport de la vingt-huitième session : 29 juin- 14 juillet 1988 : ancienne Olympie. -- [Lausanne] : Comité International Olympique; [Athènes] : Comité Olympique Hellénique, [1988]. - 311 p. : il.

* Academia Olimpica Española. Sesión (22à : 1989 : Granada)

Academia Olfmpica Española : XXII sesión XX aniversario. — [Madrid] : Comite Olfmpico Español, DL 1990. - 235 p. : il.

* Le Comité International Olympique / mise à jour sous la direction de M. Raymond Gafner par Marie-Hélène Roukhadzé. — [2è. ed.]. —Lausanne : le Comité, cop. 1990. - 17 I p. : il.

* Comité Olímpico Internacional. President (1952-1968: Brundage) Adresses of president Avery Brundage at solemn openings of IOC sessions between 1952 and 1968. - [Lausanne] : Comité International Olympique, [196-?]. - $102 \mathrm{p}$.

* Durántez, Conrado

La Academia Olimpica Internacional / [Conrado Durántez]. - [Madrid] : Comite Olímpico Espafol, 1988. - 172 p. : il.- ISBN 84-404-3163-5

* Mayer, Otto

A través de los aros olímpicos : historia del Comité Olímpico Internacional y de sus sesiones desde 1894 a 1960 : una serie de documentos auténticos, algunos de ellos inéditos / Otto Mayer. - [Madrid] : Comité Olímpico Español, DL 1962. - 309 p. : il. - (Olimpia)

Richard, $\mathrm{V}$.

Athenes : l'ancienne olympic et l'Academie Olympique / V. Richard. - Ortawa : University of Ottawa, 1975. - I9p.

Silance, L.

Comite Olimpico Internacional : a sua evoluçao juridica : passada, presente, futuro / L. Silance. - Lisboa : Ministerio da Educaçao e Cultura. Direcçao-Geral dos Desportos, $1986 .-28 \mathrm{p}$.

\subsection{Carta Olimpica}

Olympic charter 1982. — Lausanne : Comité International Olympique, 1982. $145 \mathrm{p}$.

Olympic charter 1984. - Lausanne : Comité International Olympique, cop. 1983. $-159 \mathrm{p}$. 
Olympic charter 1985. - Lausanne : International Olympic Commitree, cop. 1985. - $115 \mathrm{p}$.

* Olympic charter 1987. - Lausanne : International Olympic Committee, [1987]. $-60 \mathrm{p}$.

* Olympic charter 1989. - Lausanne : International Olympic Committee, [1989]. $-60 \mathrm{p}$.

* Carta olímpica 1990. - Lausanne : Comité Olimpico Internacional, [1990]. $67 \mathrm{p}$.

\subsection{Diversos}

* Durántez, Conrado La Antorcha olímpica : el gran símbolo olímpico / [Conrado Durántez]. - [Madrid] : Comité Olímpico Español, 1987. - 195 p. : il. - ISBN 84-505-4896-9

* Foorman, Vsevolod

Olympic stamps / Vsevolod Foorman. - Moscow : Progress, cop. 1981. - 195 p.: il.

Hoobing, $\mathrm{R}$.

The 1984 olympics: Sarajevo and Los Angeies / R. Hoobing. - Washington D.C. : U.S. Postal Service, 1984. - 132 p. : il.

Olympex' 88 : olympic stamp exhibition = Olympex' 88 : exposition olympique de philatelie. - [Calgary] : Olympic Arts Festival, 1988. - 51 p. : il.

* Philatelie olympique = Olympic philately. $-[S . I$.$] : International Olympic Com-$ mittee, DL 1982. - [32] p. ; il.

* Soler Vilá, José Ma

Historia de los juegos olímpicos : deporte y filatelia / José Ma Soler Vilá, —Madrid : Instituto Nacional de Educación Física y Deportes, DL 1977. — [22] p. : il.

* Soler Vilá, Jose $\mathrm{M}^{2}$

Sellos olímpicos : [1896-1966] / José Ma Soler Vilá. — Madrid : Comité Olímpico Español, 1967. — 294 p. : il.

Winternheimer, $M$.

Spezialkatalog poststempel ganzsachen und sonder-einschreibzettel zu den olympischen spielen $1976 / \mathrm{M}$. Winternheimer. - Schwabenheim : Olympiaden und Sport, 1977. - 215 p. : il.

\section{HISTORIA}

\subsection{Història general}

* Brohm, Jean-Marie

Le Mythe olympique / Jean-Marie Brohm. - Paris : Christian Bourgois, cop. 1981. - 476 p. - ISBN 2-267-00248-5 
* Costelle, Daniel

Histoire des jeux olympiques / Daniel Costelle ; avec Monique Berlioux. - Paris : Pathé-TF1 : Larousse, 1980 - 238 p. : il. - ISBN 2-03-518103-8

* Finley, M.I.

The Olympic games : the first thousand years / M.I. Finley, H.W. Pleket. - London : Chatto \& Windus, 1976. - XV, I38 p. : il. - ISBN 0-7011-2087-8 Killanin, L.

The Olympic games / L. Killanin, J. Rodda. - Rev, ed. - London : Macdonald and Jane's, $1979 .-319 \mathrm{p}$. : il.

* Lagorce, Guy

La Fabuleuse histoire des jeux olympiques / Guy Lagorce, Robert Pariente. Paris : Natham, cop. 1988. - 727 p. : il. - ISBN 2-09-286437-8

* Mandell, Richard D.

Sport : a cultural history / Richard D. Mandell. - New York : Columbia University Press, cop. 1984. - 340 p. : il. — ISBN 0-231-05470-X.

Wallechinsky, D.

The Complete book of the olympics / D. Wallechinsky. - 2nd ed. - New York : Penguin Books, cop. 1988. - XXIX, 687 p. : il.

\subsection{Història antiga}

\section{* Durántez, Conrado}

Olimpia y los juegos olimpicos antiguos / Conrado Durántez. — [Madrid] : Delegación Nacional de Educación Fisica y Deportes : Comité Olímpico Español, 1975. - 2 v. : il. - ISBN 84-400-9015-3

* The Glory of Olimpia : art, heroes and myths of the ancient olympic games / Carmine Ampolo ... [et al.] ; edited by Antonio Gnoli. — Milan : Arnoldo Mondadori, cop. $1985 .-158$ p. : il.

* Les Jeux olympiques dans l'antiquite / Elie Fallu ... [et al.] . - Montreal : Editions Paulines \& Apostolat des Editions, cop. 1976. - ISBN 0-88840-574-X

* Lennartz, Karl

Kenntnisse und vorstellungen von Olympia und den olympischen spielen in der zeit von 393-1896/ Karl Lennartz. - Schorndorf : Karl Hofmann, cop. 1974. -224 p. : il. - (Theorie der leibeserziehung; 9)

* Olivová, Vera

Sports and games in the ancient world / Vera Olivová. — London : Otbis, cop. 1984. - 207 p. : il. - ISBN 0-85613-273-X

* The Olympic games in ancient Greece : ancient Olympia and the olympic games I general supervision Nicolaos Yalouris. - Athens : Athenon, cop. 1982. - 303 p. : il.

* Young, David C.

The Olympic muyth of greek amateur athletics / by David C. Young. - Chicago : Ares, $1985 .-202$ p. --ISBN 0-89005-523-8 
"Papers»: Revista de Sociologia

\subsection{Història contemporània}

\section{* Gherarducci, Mario}

Olimpia amore mio : storia e personaggi dei giochi olimpici moderni dal 1896 ai giomi nostri/Mario Gherarducci. - Legnano : Landoni, cop. 1984. - 322 p. : il.

Killanin, L.

Olympic games : 80 years of people, events and records / L. Killanin, J. Rodda. - [S.I.] : Collier Macmillan Canada, 1976. - 272 p.

* Lucas, John

The Modern olympic games / John Lucas. - South Brunswich [etc.] : A.S. Barnes; London: Thomas Yoseloff, cop. $1980 .-242$ p. : il. - ISBN 0-49802447-4

* Mandell, Richard D.

Las Primeras olimpiadas modernas : Arenas, $1896 /$ Richard D. Mandell. - Barcelona : Ediciones Bellaterra, cop. 1990 . — 171 p. : il. — ISBN 84-7290-061-4

* Pujadas, Xavier

L'altra olimpiada : Barcelona'36 : esport, societat i política a Catalunya (19001936) / Xavier Pujadas, Carles Santacana . - Barcelona : Llibres de l'Índex, 1990. - 251 p. : il. - ISBN 84-87561-00-4

* Sprint, Miroir

L'Épopée des jeux olympiques : 1896-1988 / Miroir Sprint. - [S.1.] : Messidor, DE 1988. — 217 p. : il. - ISBN 2-209-06059-1

\section{OLIMPIADES I JOCS OLIMPICS MODERNS}

4.1. Jocs d'estiu

* Gueorguiev, Nikolay

Analyse du programme olympique (des jeux de P'olympiade) / etude élaborée par Nikolay Gueorguiev. - Lausanne : Maison Olympique, 1987. - 465 p.

- Edició no comercial

XVII : Roma 1960

* XVIIe olympiade Rome 1960. - [S.I.] : Banca Commerciale Italiana, [1960?]. - 3 v. : il.

* The Games of the XVII olympiad Rome 1960 : the official report of the Organizing Cornmittee. - [Rome] : Organizing Committee of the Games of the XVII Olympiad, cop. $1960 .-893$ p. : il.

XVIII : Tokio 1964

* Games of the XVIII olympiad Tokyo 1964 : official report of the Organizing

Committee. - Tokyo: Organizing Committee, 1966. - 2 v. : il. 
* Los Juegos de la XVIII olimpiada de Tokio 1964 / [dirección y redacción Frederick Fliegner]. - Lausanne : Instituto Olimpico Internacional, cop. 1965. -358 p. : il.

$X I X:$ Mèxic 1968

* Mexico 68 [Official report of the Organizing Committee]. - Mexico D.F. : Organizing Commirtee of the Games of the XIX Olympiad, cop. 1969. -$2 v_{.}:$il.

* Programa general por días : México 68 Oct-12-27, juegos de la XIX olimpiada. - México D.F. : Comité Organizador de los Juegos de la XIX Olimpiada. Departamento de Publicaciones, cop. 1968. - 1 v. (sense paginar)

\section{$X X$ : Munic 1972}

* Die Spiele : le rapport officiel du Comité Organizateur des jeux de la XXe olympiade Munich 1972. - München : Organisationskomitee für die Spiele der XX Olympiade, cop. 1974. - $3 \mathrm{v},: \mathrm{il}$.

Hass, $\mathrm{O}$.

Official guide to the games of the XXth olympiad Munich 1972/ O. Haas, U. Kaiser. - Munich: Organizing Committe, 1972. - 236 p.

Munchen 1972 : das olympische jugendlager und karte wettkampfstatten $=\mathrm{Mu}$ nich 1972 : the olympic youthcamp and map of competition sites = Munich 1972 = le camp olympique de la jeunesse et carte des terrains de competitions. — Munich : Organizing Committee, I972. - 48 p.

XXI : Mont-real 1976

Articles olympiques officiels : licensed olympic merchandise. - Mont-real : COJO, 1976. - $76 \mathrm{p}$.

* Montréal 1976 : jeux de la XXIe olympiade Montréal 1976 : rapport officiel. - Ottawa : COJO'76, cop. 1978. - 2 v. : if.

$X X I I$ : Moscou 1980

* Biétry, Charles

Les Jeux de Moscou / Charles Biétry, Renaud de Laborderie. — Paris : Solar, 1980. - Ill p.

Killanin, L.

Olympic games I980: Moscow and Lake Placid / L. Killanin, I. Rodda. - Rev. ed. - New York : Collier Books, I980. - 319 p.

XXIII : Los Angeles 1984

* Official report of the games of the XXIIIrd olympiad Los Angeles 1984. - [Los Angeles : Organizing Committee of the Games XXIIIrd Olympiad, 1985]. -889 p. : il. 
«Papers»: Revista de Sociologia

* Perelman, Richard B.

Report : press-radio-television operations at the games of the XXIIIrd olympiad 1 by Richard B. Perelman. - [Los Angeles : Los Angeles Olympic Organizing Committee], cop. 1984. - VIII, 406 p. : il.

Reich, K.

Making it happen : Peter Ueberroth and the 1984 olympics / K. Reich. - Santa Barbara : Capra Press, cop. $1986 .-274$ p. : il.

Ueberroth, $\mathbf{P}$.

Made in America : his own story / P. Ueberroch, R. Levin, A. Quinn. - New York : Morrow, cop. 1985 . -401 p. : il.

XXIV : Seïl 1988

* Official report : organization and planning. - [Seoul] : Seoul Olympic Organizing Committee, 1989. - 864 p. : il.

* Seh-Jik, Park

The Stories of Seoul olympics / by Park Seh-Jik. - Chosum-Ilbo : l'autor, cop. $1990 .-\mathrm{V}, 166 \mathrm{p}$.

* Toward one world beyond all barriers : the Seoul olympiad anniversary conference. - [Seoul] : Committee of SOAC, 1990. - $3 \mathrm{v}$.

\subsection{Jocs d'hivern}

* Gueorguiev, Nikolay

Analyse du programme des jeux olympiques d'hiver / étude élaborée par Nikolay Gueorguiev, -- Lausanne : Maison Olympique, 1988-1990. - 3 v. - Edició no comercial

* Lyberg, Wolf

The Book of facts on the olympic winter games / a study made by Wolf Lyberg. — [Stockholm : The Swedish Olympic Commiree, 198-?] - 3 v. — Edició no comercial

X: Grenoble 1968

* Taillandier, Jean-Pierre

Les Jeux olympiques d'hiver : Grenoble 1968/Jean-Pierre Taillandier, Robert Chastagnol. - Paris : Transavia-Equipment, $1967 .-192$ p. : il.

\section{$X I$ : Sapporo 1972}

* Official guide book Sapporo olympic winter games 1972 = Guide officiel des jeux olympiques d'hiver de Sapporo 1972. - Sapporo: Organizing Committee, 1972 . $-235 \mathrm{p}$.

Sapporo olympic winter games : official programme = Jeux olympiques d'hiver : programme officiel. - Sapporo: Organizing Committee, 1972. - $1 \mathrm{v}$. 
Bibliografia sobre olimpisme

Sapporo olympic winter games 1972. - Tokyo : Jitsugyo No Nihon Sha, 1972. $-189 \mathrm{p}$.

XII: Innsbruck 1976

* Mercé Varela, A.

Juegos olímpicos 1976 : Montreal, Innsbruck 1976 / A. Mercé. - Barcelona: Círculo de Lectores, 1976. - 207 p. : il. — ISBN 84-2260-822-7

Offizieller fuehrer durch die XII olympischen winterspiele, 4-15 februar 76 Innsbruck official programme. - [S.1.] : Arge Olimpia, cop. 1976. - 178 p.

XIII : Lake Placid 1980

Baker, E.H.

XIII Olympic winter games, Lake Placid 1980/ E.H. Baker, D. Baker. - Chicago : [s.n.], cop. 1979. - XXV, $230 \mathrm{p}$.

Grube, $F$.

Grosse olympiabuch : Lake Placid 1980 : dokumentation, bilanz, analyse / F. Grube, G. Richter. - Hamburg : Hoffman und Campe, 1980. - 191 p.

Report on the 1980 winter olympic games at Lake Placid. - New York : Senate, 1980. - $39 \mathrm{p}$.

* Spallek, E.P.

Olympic winter games, USA 1980 / E.P. Spallek. -- [New York] : E.P. Spallek, cop. 1980. - [223] p. : il.

\section{XIV : Sarajevo 1984}

Olympic games 1984 official report. - London : British Olympic Association, [1985?]. - 132 p. : il.

Sarajevo 1984 : das F.A.Z.-Olympiabuch / heratsgegeben von der Sportredaktion der Frankfurter Allgemeinen Zeitung. - Bad Homburg : Limpert, 1984. 159 p. : il. - ISBN 3-7853-1430-3

\section{XV: Calgary 1988}

Facts and information : Calgary 1988 olympic winter games = Faits et renseig . nements : Calgary 1988 jeux olympiques d'hiver. - Calgary : XV Olympic Winter Games Organizing Committee, 1987. — 51 p. : il.

\section{OLIMPISME I MITJANS DE COMUNICACIO DE MASSES}

* Andreff, Whadimir

Le Sport et la télévision : relations économiques : pluralite d'intétêts et sources d'ambiguittés / Wladimir Andreff et Jean-François Nys avec la collaboration de Jean-François Bourg. - Paris : Dalloz, DL 1987. - 158 p.

* Guide des media = Media guide. - [Lausanne] : Comitế International Olympique, cop. 1990 . - 189 p. : il. 
* Media, sports \&c society / edited by Lawrence A. Wenner. — Newbury Park [etc.] : Sage, 1989. - 315 p. - ISBN 0-8039-2826-2

* Minimum requirements for the electronic, written and photographic coverage and broadcasting by radio and television of the games of the olympiad and the olympic winter games. - [Lausanne] : International Olympic Committee, 1985. - 149 f. - Edició no comercial

Moragas i Spà, Miquel Jocs olimpics i comunicacio / Miquel Moragas i Spà (monogtafia en vies de publicació)

* The Olympic movement and the mass media : past, present and future issues. — Calgary [etc.] : Hurford, cop. 1989. - XX, 390 p. (paginació múltiple). ISBN 0-88953-115-3

* Perelman, Richard B.

Report : press-radio-television operations at the games of the XXIIIrd olympiad I by Richard B. Perelman. - [Los Angeles: Los Angeles Olympic Organizing Committee], cop. 1984. - VIII, 406 p. : il.

* Symposium International Sport Medias Olympisme. (1è : 1984 : Iausana) Sismo 84 : rapport officiel : official teport. — [Lausanne : s.n., 1984?]. - 275 p. : il.

\section{6.- OBRES COMPI,EMENTARIES}

\subsection{Medicina}

* Comité Olímpico Internacional. Comisión Médica

Un Développement positif $=$ A positive development $=\bigcup_{n}$ desarrollo positivo / A. Dirix [preparè par]. - Lausanne : Comité International Olympique, cop. 1984. $-47 \mathrm{p}$.

* Dopaje : sustancias prohibidas, especialidades farmacéuticas que las contienen y productos alternativos. - Madrid : Ministerio de Educacion y Ciencia. Consejo Superior de Deportes, DL 1990. - 31 p.

* Libro olimpico de la medicina deportiva / [dirigido por] A. Dirix, H.G. Knuttgen, K. Tittel ; revisión de la versión española Ramón Balius i Juji, Ferran A. Rodríguez i Guisado. - Barcelona : Doyma, DL 1990. - XVIII, 733 p. : it. - (Enciclopedia de la medicina deportiva ; 1). - ISBN 84-7592-341-0

* Noret, André

Le Dopage / Andre Noret. — Paris : Vigot, 1981. - 326 p. - ISBN 2-71140810-8

\subsection{Entrenament}

* Platonov, Vladimir Nikolaievich

La adaptación en el deporte / por Vladimir Nikolaievich Platonov; revisado y adaptado por Roser Coll. - Barcelona : Paidotribo, DL 1991. - 313 p. (Deporte \& Entrenamiento). - ISBN 84-86475-73-2 


\subsection{Psicologia}

\section{* Alderman, Richard B.}

Manuel de psichologie du sport / Richard B. Alderman; traduit de l'édition américaine par Jeanne Etoré. - Paris : Vigot, 1987. - 307 p. - (Sport + enseignement ; 68). - ISBN 2-71 14-0866-3

* Fernández, Luis

Savoir gagner : la réussite en compétition / Luis Fernández. — Paris : Amphora, 1989. - 198 p. - (Savoir-faire sportif). - ISBN 2-85180-164-3

* Thomas, Raymond

La Psychologie du sport de haut niveau / Raymond Thomas, Guy Missoum, Jean Rivolier. - Paris : Presses Universitaires de France, 1987. - 300 p. -(Pratiques corporelles). - ISBN 2-13-039842-1

\subsection{Esport i societat}

\section{* Bourg, Jean-François}

Le Sport en otage / Jean-François Bourg. — Paris : La Table Ronde, cop. 1988. - 224 p. - ISBN 2-7103-0359-0

\section{* García Ferrando, Manuel}

Aspectos sociales del deporte : una reflexión sociologica / Manuel Garcfá Ferrando.

- Madrid : Alianza Editorial : Consejo Superior de Deportes, cop. 1990. -323 p. - (Alianza Deporte). - ISBN 84-206-5703-4

* Mandell, Richard D.

Historia cultural del deporte / Richard D. Mandeil. - Barcelona : Bellaterra, cop. 1986. - 346 p. : il. — ISBN 84-7290-047-9

* Mazeaud, Pierre

Sport et liberté / Pierre Mazeaud. - Paris : Denoël, cop. $1980 .-230$ p.

* McInstosh, Petet Sport in sociery / Peter McIntosh. — [ed. rev. 1987]. — Twickenham : West London Press, 1987. - 236 p. - ISBN 0-9507243-5-1

* Sport, culture and ideology / edited by Jennifer Hatgreaves. — London [etc.] : Routledge \& Kegan Paul, 1982. — IX, 254 p. — ISBN 0-7100-9242-3

\subsection{Economia / Politica}

* Five-ring circus: money, power and politics at the olympic games / edited by Alan Tomlinson and Garry Whannel. - London [etc.] : Pluto Press, 1984. $\mathrm{X}, 116$ p. - ISBN 0-86104-769-9

* Kanin, David B.

A Political history of the olympic games / David B. Kanin. - Boulder : Westview Press, 1981. - XIV, 161 p. - ISBN 0-86531-109-9 
«Papers»: Revista de Sociologia

* Klatell, David A.

Sports for sale : television, money, and the fans / David A. Klatell, Norman Marcus. - New York [etc.] : Oxford University Press, 1988. — VIII, 253 p. ISBN 0-19-503836-3

* Shaikin, Bill

Sport and politics : the olimpics and the Los Angeles games / Bill Shaikin. New York [etc.] : Praeger, 1988. - X, 105 p. - ISBN 0-275-92786-5

* Sport and international relations / edited by Benjamin Lowe, David B. Kanin, Andrew Strenk, - Champaign : Stipes, cop. 1978. - XV, 627 p. - ISBN $0-87563-162-2$.

\subsection{Dret}

* 1992 et le sport / études publiées sous la direcrion de Luc Silance. - Brugge : La Chartre : 1992 Secretariat d'Etat a l'Europe, 1991. - XI, 192 p. - ISBN 90-6200-46I-X

* Berthelot, Daniel

Statut social et fiscal des athletes de haut niveau / Daniel Berthelot, Thierry Lamorlette, Raphaële Sablayrolles. - Paris : Economica, cop. 1989. - IX, 100 p. - (Audit-Gestion-Fiscalité). - ISBN 2-7178-1707-7

* Espanya. Ley del deporte

Ley 10-1990 de 15 de octubre del deporte. - Madrid : Departamento de Programación Edirorial del BOE, 1990. - 54 p. - ISBN 84-340-0380-5

\subsection{Fair Play}

* Manifiesto sobre el fair play : establecido por el C.I.E.P.S. en cooperacion con el C.O.I. e [sic] con la colaboración de la U.N.E.S.C.O. - Paris : C.I.E.P.S., [198-?]. $-31 \mathrm{p}$.

\subsection{Dona i Esport}

\section{* Creff, Albert-François}

La Femme et le sport / par Albert-François Creff et Michel-François Canu. Paris [etc.] : Masson, 1984. - VIII, 14I p. — (Monographies de medicine du sport). - ISBN 2-225-75680-5

\section{BARCELONA' 92}

* 3ème Rapport d'activité = 3rd Progress report $/$ Commission de Presse ClO. Barcelona : COOB'92. Division Opérations de Presse, 1990 - 2 v. : il. - Edició no comercial 
* Los IX juegos paralímpicos Barcelona '92. -- Barcelona : Comité Organizador Olímpico Barcelona '92. División de Paralímpicos, 1990. - 25 p. - Edició no comercial

* Los IX juegos paralímpicos de Barcelona '92 : dossier informativo. - Barcelona : COOB'92. Divisió de Paralímpics, 1991. - 31 p. - Edició no comercial

* Els 16 esports dels IXns jocs paralímpics de Barcelona '92. - Barcelona : COOB'92. Divisió de Paralímpics, cop. 1990. - 12 p. - Edició no comercial

* 500 dies per als jocs olímpics $:$ dossier de premsa $=500$ días para los juegos olímpicos : dossier de prensa $=$ A $\mathbf{5 0 0}$ jours des jeux olympiques $:$ dossier de presse $=500$ days to the olympic games $:$ press dossier. - Barcelona : COOB'92, 1991. - l v. : il. - Edició no comercial

* Barcelona : la ciutat i el 92. - Barcelona : Institut Municipal de Promoció Ur. banistica : Olimpiada Cultural, DL $1990 .-305$ p. : il - ISBN 84-8764702-2

* Barcelona '92 : guia. - Barcelona : Comité Organitzador dels Jocs de la XXVa Olimpíada Barcelona 1992, 1991. - 174 p. : il. + 1 plànol. - ISBN 84-7868024-1

* Calendari de competició jocs de la XXVa olimpiada Barcelona 1992. - Barce. Iona : COOB'92, 1990. - 158 p. - ISBN 84-7868-020-9

* Cartells olímpics oficials i col.lecció de cartells de dissenyadors. - Barcelona : COOB'92, cop. 1991. - 25 p. : il.

* Les Ciutats de Barcelona '92. - Barcelona: COOB'92 : Diputació de Barcelona, DL 1991. - 144 p. : il + I plànol. - ISBN 84-7868-032-2

* Le Climat dans les sites olympiques = Climatology of the olympic sites : Barcelona '92. - Barcelona : COOB'92. Divisió d'Atenció a la Famítia Olímpica, [1990?]. - 50, 50 p. : il. - Edició no comercial

* COOB'92

Informe de progrés $=$ Informe de progreso $/$ Comissió Mèdica del COI. - Bar celona : $\operatorname{COOB}^{\prime}$ '2. Divisió d'Atenció a la Família Olímpica, [1990]. - 76, 76 p. - Edicio no comercial

* COOB'92

Pla d'assistència sanitària olímpica (PASO'92) = Plan de asistencia sanitaria olímpica (PASO'92). - Barcelona : COOB'92. Divisió d'Atenció a la Família Olimpica, 1989. - 141,141 p. : il. - Edició no comercial

* COOB'92

$\mathrm{Pla}$ director : sisena versió desembre'90 / Comitè Organitzador Olímpic Barcelona '92. - Barcelona : Comitè Organitzador Olímpic Barcelona '92, Divisió de Planificació i Control, 1990. - 2 v. : il. - Edició no comercial

* COOB'$^{2} 2$

Presentation of the COOB'92 medical services progress report to the IOC medical commission $=$ Presentation du rapport d'activité des services médicaux du COOB'92 à la comission médicale du CIO, - Barcelona : COOB'92. Divisió d'Atenció a la Família Olímpica, [1991]. - [82] f. - Edició no comercial 
* Criterios de patrocinio y colaboración empresarial en los Juegos de la XXV Olimpíada Barcelona ' 92 = Criteria for commercial sponsorship and association at the Games of the XXV Olympiad Barcelona 1992. - Barcelona : COOB'92. Divisió Comercial, 1991. - 64, 62 p. - Edició no comercial

* Cursos de formació voluntaris'92 / Comitè Organitzador dels Jocs de la XXVa Olimpiada Barcelona 1992. - 2a. ed. rev. - Barcelona : COOB'92, 1990. 266 p. en paginació múltiple. - ISBN 84-7868-014-4

* De Colonia Iulia Augusta Faventia Paterna Batcino a Barcelona '92 : algunes notes per a un millor coneixement de la ciutat, la seva evolució i la seva història. - Barcelona : COOB'92, DL 1990. - 61 p. : il. — ISBN 84-7868011-X

* L'Estadi olímpic símbol de Barcelona '92. - Barcelona : COOB'92, 1989, 44 p. : il. - ISBN 84-7868-001-2

* Guia : la Barcelona del 93. - Barcelona : Ajuntament de Barcelona. Regidoria de Relacions Institucionals, 1990 . - 130 p. : il. - ISBN 84-85905-72-5

* Guía de clasificaciones generales y funcionales : IX Juegos paralímpicos Barcelona '92. - Barceiona : COOB'92. Divisió de Paralímpics, cop. 1988. - 115 p. : il. - Edició no comercial

* Guía de Cobi animado : juegos de la XXV olimpiada Barcelona 1992 = Cobi animation guide : games of the XXV olympiad Barcelona 1992. - Barcelona : COOB'92, DL 1990. - 17 p. : il.

* Guía de medios de comunicación. - Barcelona : COOB'92. Operaciones de Prensa y Radiotelevisión. División de Paralímpicos, 1991. - 35 p. : il. - Edició no comercial

* Guia de premsa. - Barcelona : COOB'92, 1991 - 83 p. : il. + I plànol. ISBN 84-7868-028-4

* Guia preliminar de la Vila Olímpica = Guía preliminar de la Villa Olímpica. - Barcelona : COOB'92. Divisió d'Allotjament, 1990. - 27, 27 p., [8] f. : il. - Edició no comercial

* Información climatológica : Barcelona '92. - [Barcelona : COOB'92, 1991]. - 33 p. : il. - Edició no comercial

* Jocs de la XXVa olimpiada Barcelona 1992 : manual de normes gràfiques = Juegos de la XXV olimpíada Barcelona 1992 : manual de normas gráficas = Jeux de la XXVe olympiade Barcelona 1992 : manuel de normes graphiques = Games of the XXV olympiad Barcelona 1992 : graphic standars manual. - Barcelona : COOB'92, cop. 1988. - 1 v. : il. - Edició no comercial

* Jocs de la XXVa olimpíada Barcelona 1992 : variacions de la mascota = Juegos de la XXV olimpíada Barcelona 1992 : variaciones de la mascota = Jeux de la XXVe olympiade Barcelona 1992 : variations de la mascotte = Games of the XXV olympiad Barcelona 1992 : mascot variacions. - Barcelona : COOB'92, cop. 1988. - I v. : il. - Edició no comercial

* Manual de formación del voluntario olímpico. - Barcelona : $\operatorname{COOB}^{\prime} 92$. División de Voluntarios, cop. 1989. - 32 p. : iL. - ISBN 84-7868-008-X 
* Olimpíada Cultural

Olimpíada Cultural S.A. : memoria 1990 = Olimpiada Cultural S.A. : memoria 1990. - Barcelona : Olimpiada Cultural, 1991. - 91, 93, [14] p. : il. - Edició no comercial

* Olimpíada Cultural. - Batcelona : [COOB'92. Divisió de Cultura], DL 1988. - [27] p. - ISBN 84-86279-12-7

* El Palau d'esports Sant Jordi : els projectes d'Arata Isozaki / [coordinació Toshiaki Tange] ; textos Arata Isozaki ... [et al.]. - [Barcelona : Diputació de Barcelona], DL 1990. - 87, [11] p. : il. — ISBN 84-600-7327-0

* Palau Sant Jordi. - Barcelona : Diputació de Barcelona : COOB'92, 1990. -95 p. : il. - ISBN 84-7868-017-9

* Paralímpics Barcelona '92 = IXe jeux paralympiques de Barcelona ' 92 = IX paralympic games Barcelona '92. - Barcelona : COOB'92. Departament de Publicacions, cop. 1990. - [19] p. : il. - Edicí no comercial

* Las Paraolimpiadas : algo más que una competición deportiva. - Barcelona : Fundació Institut Guttmann, 1990. - 98 p. : il. - (Blocs ; 1)

* Els Pictogrames d'esports dels jocs olímpics de Barcelona ' 92 = Los Pictogramas de deportes de los juegos olírnpicos de Barcelona ' 92 = Les pictogrames des sports des jeux olympiques de Barcelona ' $92=$ The Sports pictogrammes of the Batcelona '92 olympic games. - Barcelona : COOB'92. Divisió d'Imatge i comunjcacío, DL 1990. - 75 p. : il. - Edició no comercial

* Pla d'assistència sanitària dels jocs paralimpics (ASPA'92) : IXns jocs paralímpics de Barcelona '92. - Barcelona : COOB'92. Divisió de Paralímpics, cop. 1990. -70 p. - Edició no comercial

* [Solicitud de candidatura al COI para la celebración en Barcelona de los juegos de la XXVa olimpiada]. - [S.1. : s.n., 1986]. - 6 v. + 1 cassette sonora + 1 disket +1 vídeocassette ; en contenidor. - Edjció no comercial

* Les Subseus olímpiques'92. - Barcelona : COOB'92. Coordinació Subseus Olimpiques, 1991. - [57] f. : il. - Edició no comercial

* The Wind in Batcelona during the summer months. - Barcelona : COOB'92. Divisió d'Atenció a la Família Olímpica, [1990]. — 17 p. : il. - Edició no comercial

\section{PUBLICACIONS PERIODIQUES}

\section{OLIMPISME}

* Mensaje olímpico / Comité Olímpico Internacional. - Ed. castellana. - No 1 (1982)- . - Lausanne : Comité Olímpico Internacional, 1982Quadrimestral. - Altres edicions en diverses llengües

* Revista olímpica / Comité Olímpico Inrernacional. - Ed. castellana. - Núm. 62 (1973)- - Lausanne : Comité Olímpico Internacional, 19??- 10 núm. l'any. - Altres edicions en diverses llengües 


\section{BARCELONA ' 92}

* Barcelona ' 92 info subseus : butlletí d'informació general per als Comitès de Subsetu Olímpica i els Comitès Tècnics de Subseu = Barcelona '92 info subsedes : boletín de información general para los Comités de Subsede Olímpica y los Comités Técnicos de Subsede. - Núm. I (ocrubre 1990)Barcelona : COOB'92. Coordinacío Subseus Olfmpiques, 1990- Mensual. - Edició no comercial

* Barcelona olímpica : la tevista de los juegos de 1992. - Núm I (jun. 1988). - Barcelona : Sports Editor, 1988-

10 núm. l'any, amb algunes irregularitats. - A partir del núm. 19 l'editor és: Olimpress

* Bcra'92 Newsletter. - Núm. 1 (juny 1989). — Barcelona : COOB'92. Divisió d'Imatge i Comunicació-Pretnsa, 1989. Setmanal. - Altres edicions en diverses llengües. - Edició no comercial

* Cataluña olímpica : una economía en marcha - No 1 (noviembre 1987)-No 18 (abril 1989). - Barcelona : Fundació Mediterrània, 1987-1989 Mensual

* Subseus olímpiques = Subsedes olímpicas. - Núm. 1 (setembre 1990)- [Barcelona] : Coordinació Subseus Olímpiques del COOB'92, 1990Bimensual. - Edició no comercial

*Voluntaris'92. - Núm. I (setembre 1988)- . - Barcelona : COOB'92, 1988- Periodicitat irregular. - Edició no comercial

Com a complement d'aquesta bibliografia, creiem interessant afegir les referències de les bases de dades especialitzades en esport més importants.

\section{BASES DE DADES}

$* 1$.

Nom: ALCATEL

Productor: COOB'92 i Cia. Alcatel

Contingut:És organitzada en vuit apartats:

- Història dels jocs olímpics (resum de les 24 edicions dels jocs d'estiu).

- Barcelona '92 (procés d'organització dels jocs olímpics de 1992).

- Estrelles per al 1992 (biografies d'esportistes que eventualment poden participaren els jocs de Barcelona).

- Moviment olfmpic (estructura del COI, un directori dels comités olímpics nacionals, federacions olimpiques i organitzacions internacionals reconegudes pel COI).

- Barcelona avui.

- Catalunya avui. 
- Espanya avui, (aquests tres úlcims punts proporcionen informació històrica i actual, i guies d'adreçes i teleffons).

- Butiletí de notícies (informació diària de caràcter general $i$ informació referida específicament als treballs de preparació dels jocs de 1992).

Accés: En línia, a través de qualsevol dels centres esmentats. El Projecte Alcatel fun" cionarà fins al començament dels jocs.

*2.

Nom: Punts d'Informacio Cultural (PIC)

Productor: Ministeri de Cultura

Contingut:Els PIC ofereixen tres bases de dades especialitzades en esport i olimpisme:

- DESP (informació dels Ilocs d'honor obtinguts per l'esport espanyol en campionats internacionals des de 1938).

- FUTB (partits internacionals de futbol i resultats dels partits jugats per la selecció espanyola en els campionats del món des de 1930).

- OLIM (resultats i classificacions en tots els esports durant els jocs olímpics moderns i en les Universiades).

Accés: A través de les seus instal.lades en dependències del Ministeri de Cultura i en entitats col-laboradores. A Barcelona: Carrer Mestre Nicolau, 19.

*3.

Nom: $\quad$ SPORT

Productor: Sport Information Resource Centre (SIRC)

Contingut:Aproximadament 250.000 referències bibliogràfiques (un $30 \%$ inclouen abstracts) de documents que tracten tots els aspectes rełacionats amb l'esport i les ciències de l'esport. La cobertura ternporal és: monografies i tesis des de 1949, publicacions periodiques des de 1975.

Accés: En línia a través de BRS, DLALOG, DIMDI i CAN/OLE. En els tres primers centres esmentats al començament es pot consultar la versió en CD-ROM "Sport Discus".

4.

Nom: AGORA SPORTIF

Productor: Agence France Press (AFP)

Contingut:Aproximadament 210.000 referències bibliogràftques. Principalment dóna informació sobre selcccions, competicions, rècords, palmarès i biografies de professionals de l'esport, des de 1983.

Accés: En línia a través de G.CAM SERVEUR i QUESTEL. 
"Papers»: Revista de Sociologia

5.

Nom: HERACLES

Productor: SPORTDOC

Contingut:Aproximadament 35.000 referències bibliograłfiques sobre ciències de l'educació física i l'esport, des de 1973.

Accés: En línia a través de G. CAM SERVEUR.

* 6 .

Nom: $\quad$ SPOLIT

Productor: Bundesinstitur fuer Sportwissenschaft (BISp).

Contingut:Aproximadament 55.000 referències bibliogràfiques (un $95 \%$ amb abstracts) sobre tots els aspectes de les ciències de l'esport, des de 1974. Accés: En línia a través de DIMDI. 